\title{
Algorithm Based New Tone Reservation Method for Mitigating PAPR in OFDM Systems
}

\author{
Tanmoy Saha, Shreyasi Chakrabarty, Subhankar Bhattacharjee, Sanjib Sil
}

\begin{abstract}
There are abundant methods to mitigate PAPR in OFDM signals among which algorithm based tone reservation is of great popularity owing to its low complexity as well as decent BER. Here we have put forward a new distinct algorithm based Tone Reservation technique which is not only less complex and calculates its own threshold as well as PRT signal (unlike other algorithms requiring predetermined threshold and PRT) but also aptly modifies the data by bit by bit comparison with a modified copy of itself (algorithm modified) thus scaling the peaks as and providing a decent BER and good PAPR reduction.
\end{abstract}

Keywords - BER, CCDF, OFDM, PAPR, Tone Reservation Algorithm

\section{INTRODUCTION}

O FDM is an amalgamation of modulation and multiplexing which proves to be apt and utile when using multiple carrier modulation schemes for transmission of data signals due to its high data rate. Here high rate input data gets partitioned into smaller data stream and get modulated using many sub carriers which have the property of orthogonality so that they cannot interfere with each other. The applications of OFDM goes a long way from Digital Audio Broadcasting (DAB), Wireless LAN IEEE 802.11a, IEEE 802.11g, 3G, 4G mobile phone standards to many more. All the above said applications of OFDM are a consequence of various advantages like including spectral efficiency which is very high, robustness to channel fading, immunity to impulse interference, spectral density average which is unique, capacity of handling echoes which are very strong and distortion which is non linear in nature and is very low. But despite having these advantages there are a few inferiorities among which PAPR i.e. the ratio of Peak power to average power which is of utmost importance as it causes a severe degradation in the transmitted signal thus causing errors in transmission. OFDM involves a problem PAPR which gives inter-modulation and an out-of-band radiation while travelling within non linear devices [1]. The sequences having same phase get added coherently and thus turn out to be above average. This problem is known as PAPR problem i.e. peak power of system is much higher than the average power of the system. High power amplifiers (HPA's) are used in most radio systems, e.g. Solid State Power Amplifier (SSPA), is utilized in transmitter for obtaining necessary power for transmitting signals [2]. In order to achieve maximum efficiency in output power, the operation of HPA is generally at or close to saturation area, thus introducing distortion which is memoryless and also nonlinear within the channel for communication

Authors are with the Department of Electronics and Communication in Techno India College of Technology, India (e-mail: sahatanmoy663 @ gmail.com). which is very tender to the variation of the amplitudes of signal. These amplitudes show broad variation with high (PAPR) resulting into considerable inter-modulation and an out-of-band radiation passing within nonlinear devices [1]. Large PAPR demands large dynamic range in analog to digital converters (ADC's). OFDM signals show Gaussian distribution when the number of sub- carriers is very large, stating that the peak signals occur rarely and uniform quantization is undesirable[2]. So for perfect and distortion independent amplification reduction of PAPR in OFDM system is necessary. There are many techniques suggested for PAPR reduction which includes clipping and filtering (CF) method [4]-[6], probabilistic techniques [3]-[6], and tone reservation (TR) [7]-[15]. The Clipping and filtering method incorporates the slashing or rather clipping the transmitted signals so that they can be reduced down to a level determined beforehand. After clipping filtering follows which removes the out of band radiation but peak re-growth emerges out as a consequence. For mitigating this drawback an algorithm based on iteration is suggested[4] but still in-band clipping noise still remains to be eradicated resulting into increased BER[5]. Other techniques like SLM [3] which picks up the least PAPR signal among several signals obtained by multiplication of phase sequences with data sequence after they pass IFFT. SLM schemes that are based solely on bit interleaving or scrambling change binary data bits before (QAM) application to provide symbol sequences that are alternate but side information bits need to be transmitted as a compromise thus hampering efficiency. Burst errors occur as a consequence of incorrect reception of received signals. The Tone reservation technique is amongst the most favourable and apt techniques for diminution of PAPR. The technique involves the reserving a hand full of sub- carriers among the total available sub-carriers which are used for transmitting a counter signal which counteracts the peaks of the data signal transmitted thus reducing PAPR. These are known as peak reduction tones (PRTs). Various processes are available for the generation of these PRTs including quadratically constrained quadratic optimization [7], active set approach [8], gradient method [9], [15] and clipping noise based [10]-[14]. ICF [16] also reduces PAPR to a significant level but these methods require a large number of iterations which results into remarkable complexity. It is because of these drawbacks which compelled us to devise this algorithm which calculates its own threshold values as well generates its own PRTs unlike other algorithm based techniques where one has to provide the PRT values beforehand and also the threshold value and change that factor to reduce PAPR accordingly. In this method the algorithm calculates the threshold itself which is actually a factor 1.5 multiplied to median of the input signals. Then it takes a copy of the same input signal compares each bit of the copied signal 
with the threshold value and whenever it finds that the value of the copied signal exceeds the threshold the value, then the value at that particular position gets truncated and this goes on for the entire sequence. This signal acts as the counter signal i.e. PRT signal. Then an adder is used to add these two signals to get a new signal which has all its peaks reduced thus giving a significant reduction in PAPR. As OFDM signal requires the peak power to the average power ratio (PAPR) as low as possible and this can be done if the amplitudes of the PAPR reduced signal are more or less around the average value of the signal. As the peak values occur rarely in the entire OFDM transmitted signals so the median value of the signal will be approximately around the average value of the signal. Thus taking the $1.5 *$ median value of the signal as the threshold value (cause explained later) and reducing the amplitudes of the OFDM signals which are greater than this threshold will reduce PAPR to a great extend. The details of our algorithm will be discussed in section PROPOSED TECHNIQUE BASED ON ALGORITHM. Our simulation results revealed the fact that this method is less complex and yields a significant reduction in PAPR by a factor of $6.55 \mathrm{db}$ and has a decent BER. CCDF and Spectrum are also shown for the same. The paper is divided into the following sections: PAPR and OVERALL TONE RESERVATION TECHNIQUE section includes the overall technique and PAPR and the necessary mathematical calculations related to it. PROPOSED TECHNIQUE BASED ON ALGORITHM section includes the details of our proposed algorithm and how it is different from the available algorithms of the same class. SIMULATION ANALYSIS AND EVALUATION OF PERFORMANCE section includes the details of results obtained by our technique and with corresponding graphical representations. CONCLUSUION includes what our technique states in a nut shell.

\section{PAPR AND OVERAll Tone Reservation TeChNiQue}

Consider an OFDM system with the specifications:$\mathrm{N}$ sub carriers available and data bits to be transmitted

$X^{\prime}=\left[X_{0}^{\prime}, X^{\prime}{ }_{1}, \ldots \ldots X^{\prime}{ }_{N-1}\right]$

The OFDM signals are then sampled in real time form and when they are passed through the IFFT block they are formulated as given below:-

$x_{n}^{\prime}=1 / \sqrt{N} \sum_{k=0}^{L N-1} X^{\prime}{ }_{K} \times e^{j 2 \pi n k / L N}$

Here $\mathrm{n}=0,1,2 \ldots \ldots, \mathrm{LN}-1$ and the over sampling factor is represented as $\mathrm{L}$.

Thus the PAPR of the transmitted OFDM data can be denoted by:-

$P A P R=\frac{\underset{\max }{0 \leq n \leq N-1} x^{\prime} n^{2}}{E\left[\left|x^{\prime} n\right|\right]^{2}}$

Where the average power of the OFDM data is denoted by the expectation $E\left[\left|x_{n}^{\prime}\right|\right]^{2}$ and the magnitude at a particular instance is denoted by $\left|x_{n}^{\prime}\right|$.
The technique of Tone Reservation involves reservation of a hand full of sub-carriers which are used to send a counter signal denoted by C'i.e.

$\mathrm{C}^{\prime}=\left[C^{\prime}{ }_{0}, C^{\prime}{ }_{1}, \ldots \ldots \ldots \ldots, C_{N-1}^{\prime}\right]$

So, this signal counteracts the peak values of the original signal $X$ ' thus resulting in diminution of PAPR.

Let us assume out of total $\mathrm{N}$ number of sub- carriers available tone reservation technique books $N_{r}$ number of sub- carriers for sending the counteracting signal. Thus, out of total $\mathrm{N}$ subcarriers available the signal $\mathrm{X}^{\prime}$ is transmitted through $\left(\mathrm{N}-N_{r}\right)$ number of sub- carriers and the rest are used for the transmission of C' signal. This counteracting signal $C^{\prime}$ is known as the PRTs.

Let us denote a variable $\mathrm{Z}$ as the indexes where the PRTs are present. That is:

$\mathrm{Z}=\left\{i_{0}, i_{1}, \ldots \ldots \ldots \ldots, i_{N^{\prime} r^{-1}}\right\}$

So, in order to achieve a distortion less signal it is a necessary condition that the two signals i.e. $X$ ' and C' avoid overlapping with each other. Thus we can denote a set $\mathrm{Z}$ such that among the total number of available sub- carriers $N$ ' represents the set $\mathrm{Z}=\left\{i_{0}, i_{1}, \ldots \ldots \ldots \ldots, i_{N^{\prime} r^{-1}}\right\}$ consisting of $N_{r}$ sub- carriers for $C^{\prime}$ transmission and $Z^{C}$ which is the complementary set of $\mathrm{Z}$ consisting of $\left(\mathrm{N}-N_{r}\right)$ is used for the transmission of the signal $X^{\prime}$. Thus in a nut- shell we can state that if the position falls within $Z$ then the instantaneous value of $C^{\prime}$ gets transmitted and the position falls within $Z^{C}$ then $X^{\prime}$ gets transmitted. So, mathematically this condition can be represented as:-

$X^{\prime}{ }_{K}+C^{\prime}{ }_{K}=f(x)= \begin{cases}X^{\prime}{ }_{K}, & \text { if } k \in Z^{C} \\ C^{\prime}{ }_{K}, & \text { if } k \in Z\end{cases}$

Thus, we have the new signal where the peaks are reduced and the two signals $X^{\prime}{ }_{K}$ and $C^{\prime}{ }_{K}$ clearly distinguished. Thus it can be demonstrated as:-

$x^{\prime \prime}{ }_{n}=x_{n}^{\prime}+c_{n}^{\prime}=\sum_{k=0}^{L N-1}\left(X^{\prime}{ }_{k}+C^{\prime}{ }_{K}\right) \times e^{j 2 \pi n k / N}$

Thus, the peak to average power of the new and modified signal can be represented as:-

PAPR $=\frac{\underset{0 \leq n \leq N-1}{\max _{0}\left|x^{\prime} n+c^{\prime} n\right|^{2}}}{E\left[\left|x^{\prime} n\right|\right]^{2}}$

Thus from the above equations we can draw out a conclusion that in order to reduce PAPR we have to find out the optimized value of the signal $C^{\prime}{ }_{K}$ thus we can consider the optimized $C^{\prime}{ }_{K}$ as $C^{\prime}$ (opt) which is denoted as:-

$$
\mathrm{C}^{\prime}(\mathrm{opt})=\arg _{C}^{\min } \underset{C \leq n \leq N-1}{\max }\left|\sum_{k=0}^{N-1}\left(X^{\prime}{ }_{k}+C^{\prime}{ }_{K}\right) \times e^{j 2 \pi n k / N}\right|
$$

From [14] we can infer that the equation (9) turns out to be:-

$$
\text { Subject to: }\left\|x_{n}^{\prime}{ }_{n}^{\min } E^{\prime}+C^{\prime} \times P_{n}^{\text {row }}\right\| \leq \mathrm{E},
$$


Where E' can be defined as the factor of optimization and $P_{n}^{\text {row }}$ is the $n^{\text {th }}$ row of IFFT matrix.

There exists a solution for equation (10) by convex optimization, but the method requires a severely high complexity given by $\mathrm{O}\left(N_{r} L N^{2}\right)$.

At the receiver section we have hypothesised that the length of cyclic prefix is greater than the corresponding impulse response of the channel, thus after applying FFT at the receiver we have the following equation:-

$H^{\prime}{ }_{K}\left(X^{\prime}{ }_{K}+C^{\prime}{ }_{K}\right)+N^{\prime}{ }_{K}= \begin{cases}H^{\prime}{ }_{K} X^{\prime}{ }_{K}+N^{\prime}{ }_{K} & k \in Z^{C} \\ H^{\prime}{ }_{K} X^{\prime}{ }_{K}+N^{\prime}{ }_{K}, k \in Z\end{cases}$

Here $H^{\prime}{ }_{K}$ is the FFT of the impulse response of the channel. $N^{\prime}{ }_{K}$ is the noise of the FFT which is additive. So, the necessary changes in the receiver involves decoding of the tones $k \in Z^{C}$ and ignoring PRTs. The Figure 1 represents the complete diagram for the tone reservation technique.

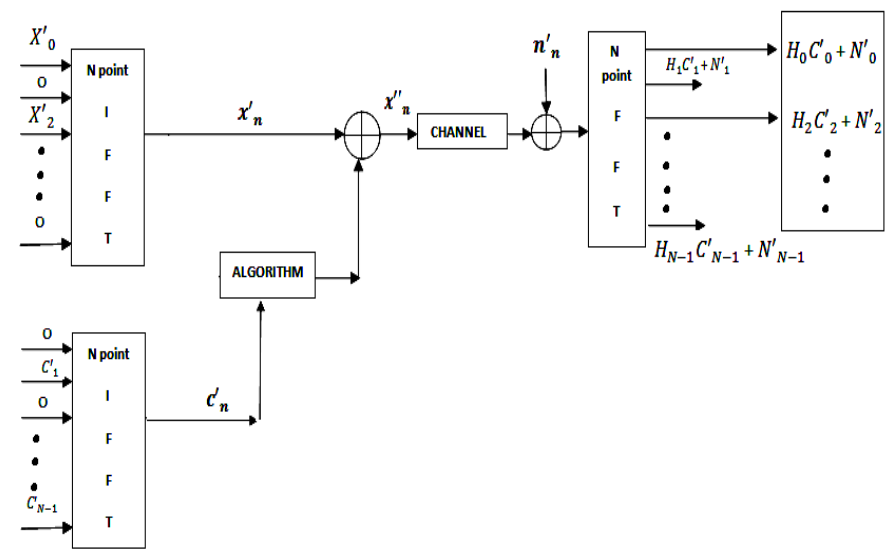

Figure 1. Tone reservation representation

\section{PROPOSED TEChNiQue BASEd ON AlgORITHM}

In the ordinary algorithm based Tone Reservation technique the counter sequence used to reduce the peak signals are provided before hand and then a threshold value which is pre determined is put forward and included into the algorithm which acts as a reference as whenever the data sequence crosses the threshold value then the value of the counter sequence i.e. $C^{\prime}{ }_{K}$ PRT is transmitted otherwise the data signal i.e. $X^{\prime}{ }_{K}$ gets transmitted. Unlike the normal technique here in our algorithm we have taken a different and contrasting approach. Instead of giving the $C^{\prime}{ }_{K}$ sequence as PRT we have considered the copy of the incoming data signal $X^{\prime}{ }_{K}$ then applied the algorithm on it and then this resulting signal after applying the algorithm is treated as the PRT or what we call it as the counter signal. Here first of all we have devised the algorithm in such a way that the algorithm will sort the entire data signal $X^{\prime}{ }_{K}$ bit by bit in an ascending order and then calculate the median value of the entire sequence. It is a well known and proven fact that in an OFDM signal the occurrence of the peak values are not regular in number. So, when we sort the entire signal in an ascending order and then calculate the median using the algorithm then it turns out that the median value of the algorithm is more or less near the average value of the signal. So instead of providing the threshold value in a predetermined way we can use this median value and multiply it with 1.5 and use this value as a threshold value. After calculating the median i.e. the threshold value the next step is to take a copy of the data signal and compare each and every bit of the copy of the data signal with the threshold value i.e. the median in our case. OFDM data signal consists of both positive and negative values and also the probability of the median being positive as well as negative is equally likely. Now here these things are taken into consideration. So, according to our algorithm the following cases arise:-

- If the median turns out to be positive then:

$>$ If the value of the data signal is positive then it is compared with threshold $=(1.5 *$ median). If it exceeds the threshold value then its value gets reduced by (Data value threshold value).

$>$ If the value of the data signal is negative then - (data value) compared with threshold $=(1.5 *$ median $)$. If it exceeds the value of threshold then its value is reduced by (Data value + median).

- If the median turns out to be negative then:

$>$ If the value of the data signal is positive then it is compared with threshold $=-(1.5 *$ median). If it exceeds the threshold value then its value gets reduced by (Data value + threshold value).

$>$ If the value of the data signal is negative then - (data value) compared with threshold $=-(1.5 *$ median $)$. If it exceeds the value of threshold then its value is reduced by (Data value - median).

Now here each bit of data of the input signal is thus compared on a bit by basis and the peak values exceeding the threshold $=(1.5 *$ median $)$ gets reduced and thus PAPR are reduced. This signal is thus considered as the counter or PRT signal denoted by $C^{\prime}{ }_{K}$. Here another important factor is taken into consideration that is we could not have used the median value only as the threshold value but if we did that then there would have been much higher chances for the loss of the information. So that's why instead of using the median alone as the threshold we have used the factor 1.5 with it. Now after applying the algorithm we have got a signal $C^{\prime}{ }_{K}$ which is the exact copy of the data signal $X^{\prime}{ }_{K}$ except that the peak values occurring in the data signal $X^{\prime}{ }_{K}$ have been reduced. Now since the two signals are same so the corresponding phases are also the same. So just before transmission we add the two signals thus forming a new signal $X^{\prime \prime}{ }_{K}$. The modified signal is formed by the following operation on a bit by bit basis:

$$
X^{\prime \prime}{ }_{K}=\left(X_{K}^{\prime}+C_{K}^{\prime}\right) / 2
$$

The reason for diving the sum by 2 is to avoid the peak re growth as a result of addition of magnitudes of the same phases. Thus this modified signal is our PAPR reduced signal desired. 
Now so according to our proposed model the algorithm is written as follows:-

\section{Algorithm 1: Tone Reservation algorithm based on median}

1. Make a copy of the data signal. Data signal = copy signal

2. Initialization: use for loop to run the loop from data bits $=0$ : final data bit.

3. Calculation of the median: by using $\mathrm{M}=$ median (copy signal).

4. Calculation of threshold: Threshold $=1.5 * \mathrm{M}$

5. Outer loop for the case of median being positive: If $(M>0)$

6. First Inner loop for the case of copy signal being positive: If (copy signal $>0$ )

7. Second inner loop for the case whether copy signal crosses threshold or not: If (copy signal > Threshold)

8. If true: Copy signal = Copy signal - Threshold

9. If false: Copy signal = Copy signal

10. Second inner loop closed

11. First inner loops else condition for the case of copy signal being negative(i.e. copy signal $<0$ )

12. Third inner loop for the case whether -(copy signal) crosses threshold or not: If ((-copy signal) $>$ Threshold $)$

13. If true: Copy signal $=$ Copy signal + Threshold

14. If false: Copy signal $=$ Copy signal

15. Third inner loop closed.

16. First inner loop closed.

17. Outer loops else condition for the case of median being negative: If $(\mathrm{M}<0)$

18. Fourth Inner loop for the case of copy signal being positive: If (copy signal $>0$ )

19. Fifth Inner loop for the case whether copy signal crosses threshold or not: If (copy signal > - Threshold)

20. If true: Copy signal $=$ Copy signal + Threshold

21. If false: Copy signal $=$ Copy signal

22. Fifth inner loop closed

23. sixth inner loops else condition for the case of copy signal being negative (i.e. copy signal $<0$ )

24. Seventh inner loop for the case whether -(copy signal) crosses threshold or not: If ((-copy signal) $>$ - Threshold)

25. If true: Copy signal = Copy signal - Threshold

26. If false: Copy signal $=$ Copy signal

27. Seventh inner loop closed.

28. Outer inner loop closed.

29. Now modified signal for transmission $=($ Copy signal + Data signal) / 2

30. End.

According to our proposed method the Tone Reservation's diagrammatic representation can be shown as in figure 2:-

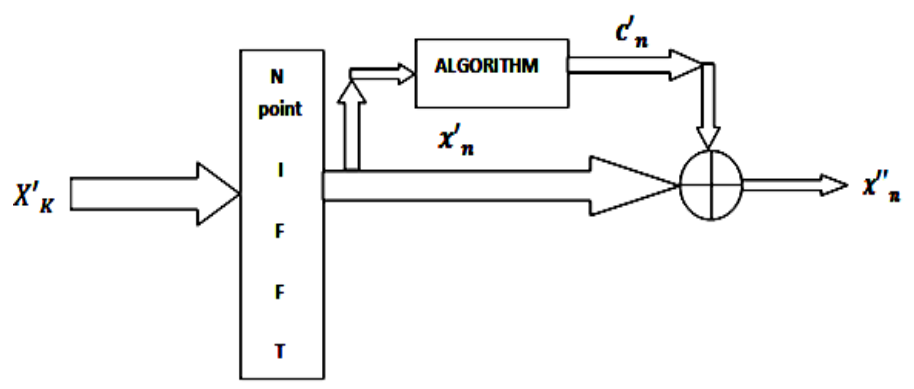

Figure 2. Tone Reservation Representation modified

\section{Simulation ANALYSIS AND EVALUATION OF PERFORMANCE}

For our method we have used 16 QAM and 256 numbers of sub- carriers along with 4 as the over sampling factor i.e. L. The figure 3 shows the transmitted signal $X^{\prime}{ }_{K}$ transmitted through $N-N_{r}$.

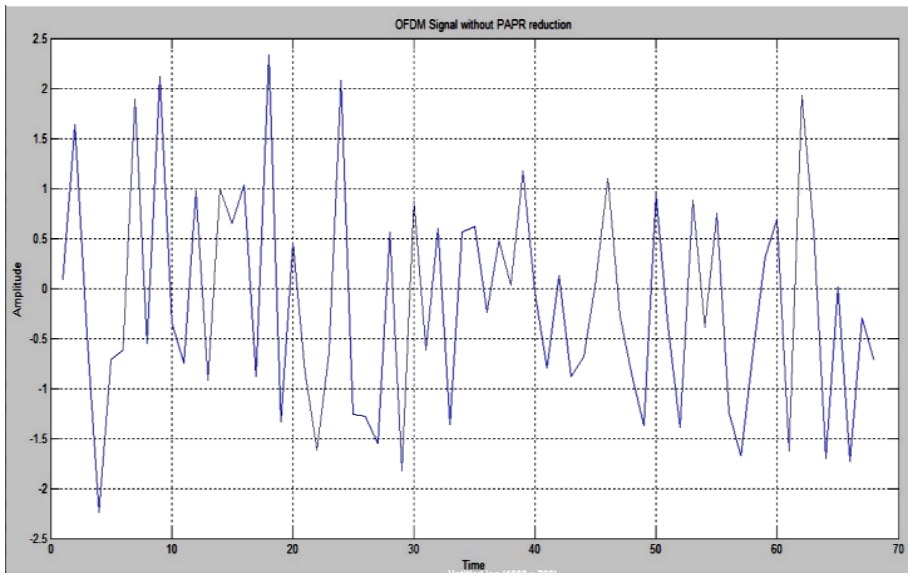

Figure 3. Representing OFDM signal without PAPR

The figure 4 denotes the PAPR reduction. Here a comparison and peak reduction is shown between the original data signal $X^{\prime}{ }_{K}$ and the modified peak reduced signal $X^{\prime \prime}{ }_{K}$. Here the blue graph represents the original signal denoted by $X^{\prime}{ }_{K}$ and the green graph represents the modified signal $X^{\prime \prime}{ }_{K}$.

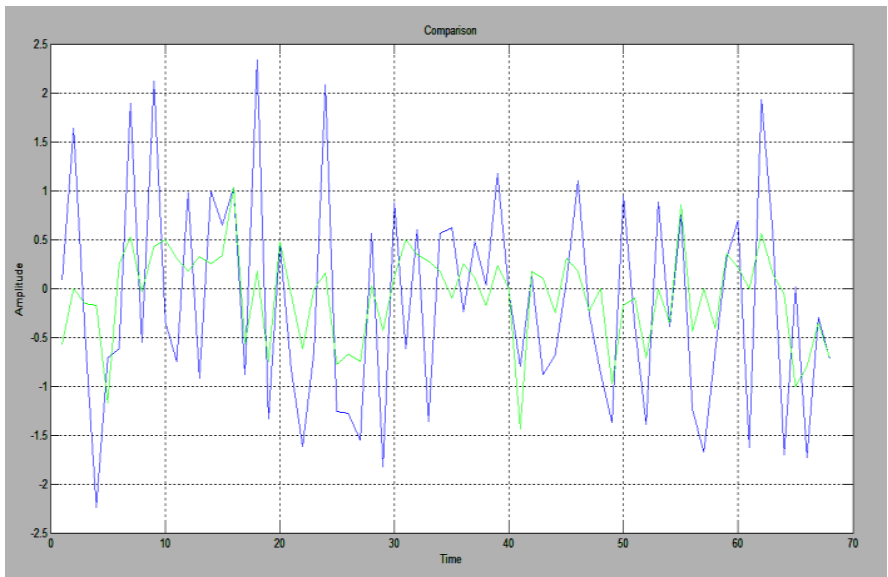

Figure 4. Comparison between original and modified signal

Below is a table named Table I representing the comparisons of various factors like 1.5 and 1 and 1 which are multiplied with the median in order to find the threshold value. By applying our proposed algorithm we have found that the PAPR of the signal gets reduced by a factor of $6.55 \mathrm{db}$ when we incorporate the factor 1.5 with median and when we incorporate the factor then a PAPR reduction of $4.9 \mathrm{db}$ is observed. However when we incorporate the factor 1 then the largest PAPR reduction i.e. $7.68 \mathrm{db}$ is observed. But a drawback is observed that is the peak values are reduced to such an extent that there is a chance of excess loss of information. So in order to get a balance between the information and the PAPR we took 1.5 into consideration while reducing PAPR of the signal. 
TABLE I

VARIATION OF THRESHOLD VALUES WITH PAPR

Value of factor Threshold value $\quad$ PAPR reduction

\begin{tabular}{ccc}
\hline 1 & Median & $7.68 \mathrm{db}$ \\
1.5 & $1.5 *$ Median & $6.55 \mathrm{db}$ \\
2 & $2 *$ Median & $4.9 \mathrm{db}$ \\
\hline
\end{tabular}

In our stated technique the receiver is involved in the detection of the data signals transmitted through the data sub- carriers i.e. $N-N_{r}$ only and the data values transmitted through the reserved sub- carriers i.e. $N_{r}$ are completely ignored. Thus our stated method neither involves changing the receiver nor does it involve exchanging data between transmitter part and receiver part. We can state the performance of our stated algorithm with the help of CCDF function i.e. Complementary Cumulative Density Function which can be mathematically represented as:-

$\mathrm{CCDF}^{\prime}\left(\mathrm{PAPR}_{0}\right)=\operatorname{Pr}\left(\mathrm{PAPR}>\mathrm{PAPR}_{0}\right)$

The CCDF of our stated algorithm is shown in figure 5.

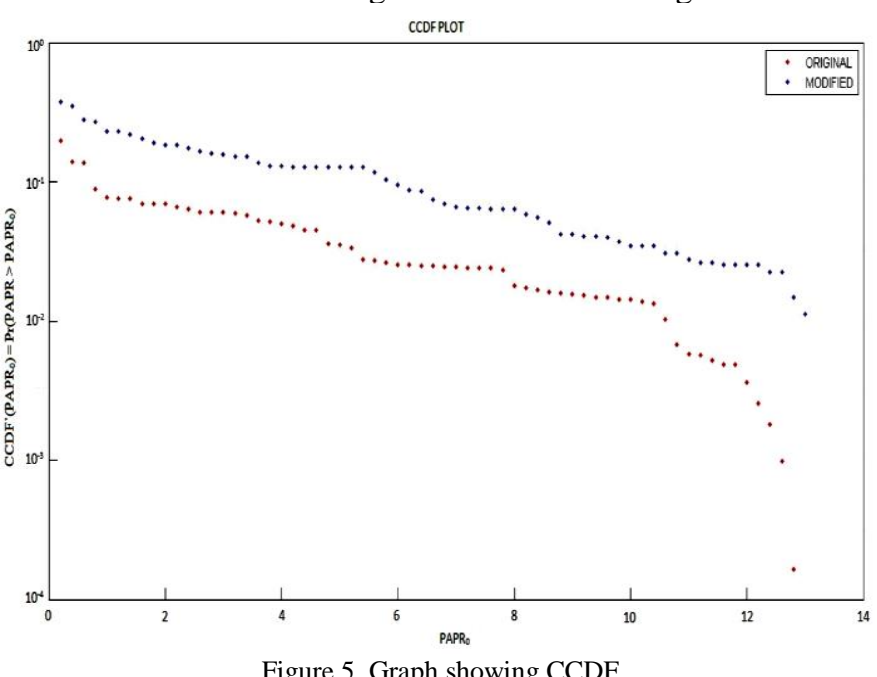

The power spectrum density function of our stated algorithm is stated in figure 6 .

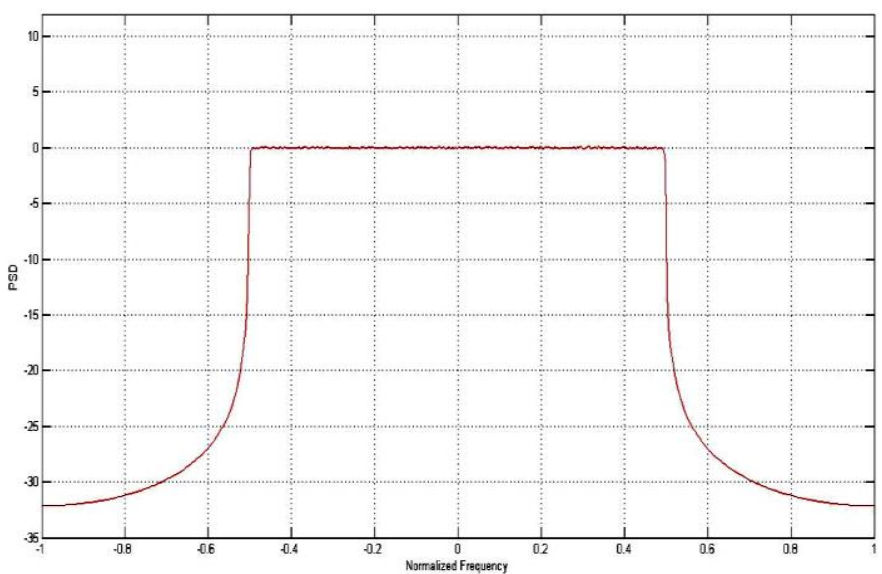

Figure 6. PSD of the modified of our algorithm
In figure 7 we have shown the BER performance of our stated algorithm based method which clearly shows that our proposed method not only maintains a good balance between the BER and SNR but also diminishes PAPR to a great extent.

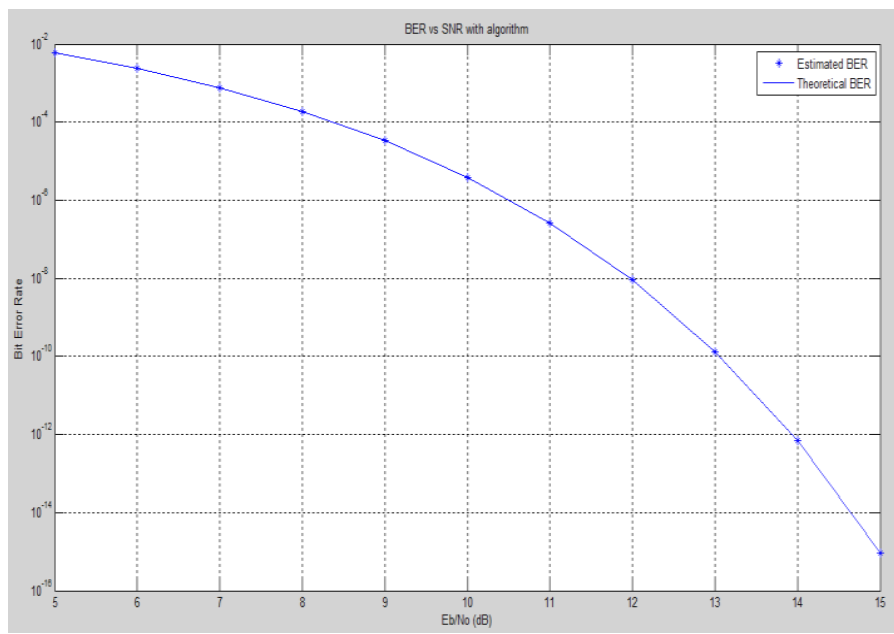

Figure 7. BER vs. SNR performance for theoretical and practical values

\section{CONCLUSION}

Finally we can conclude that amongst various available methods for diminishing PAPR algorithm based method is one of the most favorable methods as it is less complex in implementation. Among these algorithm based methods we have devised a new algorithm based method which provides its own calculates its own threshold value as well as provides its own counter signal i.e. PRT signal used for PAPR reduction which is in diametric contrast with the other available methods where one has to provide the threshold before hand as well as to provide a pre defined counter signal i.e. PRT signal for PAPR reduction. Not only this method maintains low complexity but also it provides a good PAPR reduction by a factor of $6.56 \mathrm{db}$ while maintaining a good balance between BER and SNR. Thus we can conclude that our proposed algorithm out performs other methods of the same class.

\section{REFERENCES}

[1] R. O'neal and L. N. Lopes, "Envelope variation and spectral splatter in clipped multicarrier signals," in Proc. PIMRC'95, Sep. 1995, pp. 7175

[2] M. G. Di Benedetto and P. Mandarini, "An application of MMSE predistortion to OFDM systems," IEEE Trans. Commun., vol. 44, pp. 1417-1420, Nov. 1996.

[3] M. Rodrigues and I. Wassell, "IMD reduction with SLM and PTS to impove the error probability performance of nonlinearly distorted OFDM signals," IEEE Trans. Veh. Technol., vol. 55, no. 2, pp. 537-548, Mar. 2006

[4] J. Ji and G. Ren, "A new modified SLM scheme for wireless OFDM systems without side information," IEEE Signal Process. Lett., vol. 20, no. 11, pp. 1090-1093, Nov. 2013.

[5] L. J. Cimini and N. R. Sollenberger, "Peak-to-average power ratio reduction of an OFDM signal using partial transmit sequences," IEEE Commun. Lett., vol. 4, no. 3, pp. 86-88, Mar. 2000.

[6] J. Hou, J. H. Ge, and J. Li, "Peak-to-average power ratio reduction of OFDM signals using PTS scheme with low computational complexity,"IEEE Trans. Broadcast., vol. 57, no. 1, pp. 143-148, Mar. 2011. 
[7] J. Tellado, "Peak to average power reduction for multicarrier modulation," Ph.D. dissertation, Stanford Univ., Stanford, CA, USA, Sep. 1999.

[8] B. S. Krongold and D. L. Jones, "An active-set approach for OFDM PAR reduction via tone reservation," IEEE Trans. Signal Process., vol. 52, no. 2, pp. 495-509, Feb. 2004.

[9] A. Ghassemi and T. A. Gulliver, "A simplified suboptimal algorithm for tone reservation OFDM," in Proc. IEEE ICC, Jun. 2009, pp. 1-5.

[10] J. Tellado, Multicarrier Modulation With Low PAR: Applications toDSL and Wireless. Norwell, MA, USA: Kluwer, 2000.

[11] L. Wang and C. Tellambura, "Analysis of clipping noise and tone reservation algorithms for peak reduction in OFDM systems," IEEE Trans. Veh. Technol., vol. 57, no. 3, pp. 1675-1694, May 2008.

[12] J. C. Chen and C. P. Li, "Tone reservation using near-optimal peak reduction tone set selection algorithm for PAPR reduction in OFDM systems," IEEE Commun. Lett., vol. 17, no. 11, pp. 933-936, Nov. 2010.
[13] D. Guel, J. Palicot, and Y. Louët, "Tone reservation technique based on geometric method for orthogonal frequency division multiplexing peakto-average power ratio reduction," IET Commun., vol. 4, no. 17, pp.2065-2073,Nov.2010.

[14] J. C. Chen, M. H. Chiu, Y. S. Yang, and C. P. Li, “A suboptimal tone reservation algorithm based on cross-entropy method for PAPR reduction in OFDM systems," IEEE Trans. Broadcast., vol. 57, no. 3, pp. 752-756, Sep. 2011.

[15] S. Gazor and R. AliHemmati, "Tone reservation for OFDM systems by maximizing signal-to-distortion ratio," IEEE Trans. Wireless Commun., vol.11, no. 2, pp. 762-770, Feb. 2012.

[16] J. Armstrong, "Peak-to-average power reduction for OFDM by repeated clipping and frequency domain filtering," Electron. Lett., vol. 38, no.5, pp. 246-247, Feb. 2002. 